\title{
Success With Multidisciplinary Team Work: Experience of a Primary Immunodeficiency Unit
}

Barrios $\mathrm{Y}^{1}$, Franco A ${ }^{1}$, Alonso-Larruga $\mathrm{A}^{2}$, Sánchez-Machín I ${ }^{3}$, Poza-Guedes $\mathrm{P}^{3}$, Gonzalez R ${ }^{3}$, Matheu $\mathrm{V}^{3,4}$

${ }^{1}$ Inmunología, Hospital Universitario de Canarias, San Cristóbal de La Laguna, Spain

${ }^{2}$ Bioquímica, Hospital Universitario de Canarias, San Cristóbal de La Laguna, Spain

${ }^{3}$ Alergología, Hospital Universitario de Canarias, San Cristóbal de La Laguna, Spain

${ }^{4}$ On behalf of Grupo de Estudio de Inmunodeficiencias Primarias (GEDIP) Hospital Universitario de Canarias, San Cristóbal de La Laguna, Spain

J Investig Allergol Clin Immunol 2020; Vol. 30(3): 208-210 doi: 10.18176/jiaci.0485

Key words: Multidisciplinary team. Functional study of immune response. Response to vaccines. Common variable immunodeficiency. Specific antibody deficiency.

Palabras clave: Equipo multidisciplinar. Estudio funcional de respuesta inmunitaria. Respuesta a vacunas. Inmunodeficiencia común variable. Déficit específico de anticuerpos.

Primary immunodeficiencies (PIDs) encompass a heterogeneous group of diseases caused by more than 340 genetic defects with a wide range of clinical manifestations [1]. These are grouped into broad categories based on the underlying genetic defect [2]. PIDs comprise alterations of B-cell response and B-cell lineage, alterations of the complement system, deficits in T-cell response, and the commitment of phagocyte system cells [3].

The multidisciplinary team approach usually focuses on complex conditions [4] and chronic diseases [5]. Multidisciplinary teams involve professionals from various disciplines who hold meetings to provide a more effective and efficient way of managing patients with PIDs. Multidisciplinary approaches to the management of PIDs have received little attention in the literature, probably because of the small number of cases and the specialized diagnostic approach.

In 2017, an Allergy Department was added to the care services portfolio of Hospital Universitario de Canarias (HUC), Santa Cruz de Tenerife, Spain, which provides health care to 490,000 inhabitants. Previously, autoimmunity and histocompatibility were addressed in the Immunology Department, which was the provincial reference center for kidney transplantation [6] on the island (990,000 inhabitants) and provided advice on PIDs within its area of influence.

Taking advantage of current synergies and the fact that these PIDs patients were managed by various physicians using different approaches, it was decided to create a PID Study Group (GEDIP) coordinated and directed by the Immunology Department. The GEDIP was formed by the Immunology, Allergy, Pneumology, Pediatrics, Microbiology, Internal 
Medicine, and Adult Vaccination Departments.

The group set various objectives, as follows:

(1) Design of communication protocols and rapid referral of patients to a specific care module

(2) Creation of specific dedicated diagnostic procedures to implement new diagnostic algorithms in the field of PIDs

(3) Dissemination of the group and its objectives

(4) Incorporation of new groups with synergies to improve coordination and increase efficiency

(5) Planning of training activities in the field of PIDs

(6) Communication with patient associations

An exclusive PID Unit was created consisting of 2 modules: an outpatient clinic for PIDs and a clinic for angioedema and complement disease. These modules were run within the Hospital Allergy Service but received patients from other hospital and primary care services.

During the first year, an initial meeting of the GEDIP was followed by quarterly meetings to evaluate the progress of the new unit. The diagnostic protocols implemented in the hospital immunology portfolio included the incorporation of functional studies of the immune response to conjugated protein and polysaccharide vaccines (Table).

The objectives of the GEDIP were disseminated in the hospital and in primary care (Table). In all cases, special emphasis was placed on the warning signs of immunodeficiency and the new referral channel for patients suspected of having these diseases.

In the PID module, 68 new patients were evaluated during the first year. Forty-two were women (mean age, 49.51 years; median, 55 years). Specific monthly sessions were held with the GEDIP Coordinator (Immunology) and the manager of the PID Module (Allergy) before and after the patient's visit to channel specific cases.

Most patients were from the Respiratory Medicine Department, with 22 patients, followed by Allergy with 18 patients, Hematology with 13, Primary Care with 5, Rheumatology with 3, Pediatrics with 3, Internal Medicine with 2, and ENT with 2. The main causes of consultation were recurrent bronchitis, bronchiectasis, hypogammaglobulinemia, rhinosinusitis, and pneumonia [7].

Of the 68 patients studied, 20 had allergy as their principal diagnosis (10 with asthma). Eight patients had transient hypogammaglobulinemia, 2 had $\alpha-1$ antitrypsin deficiency, and 10 had no conclusive diagnosis.

Twenty-eight patients were evaluated in the second step of diagnosis of humoral deficiencies [8], including assessment of the production of specific antibodies (diphtheria and tetanus), quantitative immunoglobulin levels and IgG subclasses, postvaccine response, monoclonal proteins, complement CH50, and lymphocyte subpopulations including T, B, and NK cells.

During this first year, 3 patients were diagnosed with common variable immunodeficiency (CVID) [9], as follows: a 32-year-old man with recurrent pneumonia; a 29-year-old woman with recurrent bronchitis; and a 38-year-old woman with an episode of severe pneumonia who was admitted to the Intensive Care Unit and had experienced recurrent respiratory infections. A fourth patient, a 68 -year-old woman with recurrent bronchitis, CVID, and thymectomy for thymoma 2 years earlier, was diagnosed with Good syndrome. Finally, 2 patients were diagnosed with specific antibody deficiency [10], namely,

\section{Table. Actions During the First Year of the PID Unit}

Design of communication and quick referral protocols

Exclusive outpatient PID module

Shared-use folder in the hospital intranet program created by the Immunology Department

Set-up of a multidisciplinary PID group (GEDIP)

Immunology, Allergy, Respiratory Medicine, Pediatrics, Microbiology, Internal Medicine, and the Adult Vaccination Module

Quarterly meetings of the GEDIP group

Monthly specific sessions of the GEDIP Coordinator (Immunology) with the PID Unit (Allergy)

A patient circuit for replacement therapy at the Day Hospital

Implementation of specific dedicated diagnostic procedures with new diagnostic algorithms in the field of PID

Functional studies of immune response to $\mathrm{T}$ cell-dependent and $\mathrm{T}$ cell-independent vaccines

Implementation of advanced lymphocyte subpopulation study including T, B, and NK cells.

Dissemination of the group and its objectives

General presentation in the Hospital Universitario de Canarias and individualized presentations in specific services

Presentations at a primary care allergy forum ( $>100$ registered GPs) and regional pediatrics meeting

Incorporation of new groups with synergies that improve coordination and increase efficiency

ENT and Hematology Departments were incorporated to GEDIP

Planning of specific training activities in the field of PID

Clinical Immunology Society and American Academy of Allergy, Asthma and Immunology

Specific training on the preparation of diagnostic reports given by the Spanish Society of Immunology

Communication with patient associations

Explicit support from the National Patient Association (AEDIP) for the Multidisciplinary Unit

Maintaining a direct information channel with the National Patient Association (AEDIP) 
a 64-year-old woman with episodes of recurrent respiratory infections and lymphoma 5 years previously and a 59-year-old woman with recurrent rhinosinusitis.

Most of the initial objectives set with the creation of the GEDIP group were fulfilled within the first year. The work of the group members has provided a new clinical perspective with respect to patients with PIDs in our setting, thus demonstrating that the interdisciplinary approach is fruitful. In the future, and with the support of the appropriate structures, the group will be reevaluated to provide adequate care for patients in this area. The inclusion of a clinical immunologist should be a key step.

Before the creation of the GEDIP, management of these patients was poorer than in other health services that are less fragmented than in the Canary Islands. In addition, previous problems included the lack of appropriate referral from primary care and a standard approach in affected patients. These problems have been resolved with the creation of the PID Unit.

The implementation of immune function studies in the response to vaccines clearly demonstrates our progress in the diagnosis achieved. Future adaptation of the referral protocols and the implementation of an advanced diagnosis pathway will improve the quality of life of patients with PIDs.

\section{Acknowledgments}

We are grateful to the Grupo de Estudio de Inmunodeficiencias Primarias (GEDIP) from Hospital Universitario de Canarias, in particular, the following departments: Pneumology (I Suarez-Toste), Microbiology (T Delgado-Melián), Preventive Medicine (J Duque-Arimany), Pediatrics (C. Martínez-Faci, V Velasco), Internal Medicine (MC Durán, A. Martínez-Riera), Ear-Nose-Throat (G de Lucas, A. Perez-Orribo), Hematology (P Machado, T MartínSantos), Immunology (Y Barrios, A Franco), and Allergy (V Matheu, I Sanchez-Machin, P Poza-Guedes, R Gonzalez, C Alava, E Mederos).

\section{Funding}

This study was funded by Hospital Universitario de Canarias, Servicio Canario de Salud.

\section{Conflicts of Interest}

The authors declare that they have no conflicts of interest.

\section{References}

1. Parvaneh N, Casanova JL, Notarangelo LD, Conley ME. Primary immunodeficiencies: a rapidly evolving story. J Allergy Clin Immunol. 2013;131:314-23.

2. Bousfiha A, Jeddane L, Picard C, Ailal F, Bobby Gaspar H, Al-Herz W, et al. The 2017 IUIS Phenotypic Classification for Primary Immunodeficiencies. J Clin Immunol. 2018;38:12943.

3. Modell V, Orange JS, Quinn J, Modell F. Global report on primary immunodeficiencies: 2018 update from the Jeffrey Modell Centers Network on disease classification, regional trends, treatment modalities, and physician reported outcomes. Immunol Res. 2018;66:367-80.

4. Pillay B, Wootten AC, Crowe H, Corcoran N, Tran B, Bowden $P$, et al. The impact of multidisciplinary team meetings on patient assessment, management and outcomes in oncology settings: A systematic review of the literature. Cancer Treat Rev. 2016;42:56-72.

5. Ferman $M$, Lim $A H$, Hossain $M$, Siow GW, Andrews JM. Multidisciplinary team meetings appear to be effective in inflammatory bowel disease management: an audit of process and outcomes. Intern Med J. 2018;48:1102-08.

6. https://www3.gobiernodecanarias.org/sanidad/scs/organica. jsp?idCarpeta=3da5f513-541b-11de-9665-998e1388f7ed

7. Yazdani $R$, Abolhassani $H$, Asgardoon $M H$, Shaghaghi $M$, Modaresi M, Azizi G, et al. Infectious and Noninfectious Pulmonary Complications in Patients With Primary Immunodeficiency Disorders. J Investig Allergol Clin Immunol. 2017:27:213-24.

8. Bonilla FA, Khan DA, Ballas ZK, Chinen J, Frank MM, Hsu JT, et al. Joint Task Force on Practice Parameters, representing the American Academy of Allergy, Asthma \& Immunology; the American College of Allergy, Asthma \& Immunology; and the Joint Council of Allergy, Asthma \& Immunology. Practice parameter for the diagnosis and management of primary immunodeficiency. J Allergy Clin Immunol. 2015;136:1186205.

9. Yazdani R, Habibi S, Sharifi L, Azizi G, Abolhassani H, Olbrich $P$, et al. Common Variable Immunodeficiency: Epidemiology, Pathogenesis, Clinical manifestations, Diagnosis, Classification and Management. J Investig Allergol Clin Immunol. 2020;30: in press.

10. Vigano S, Trabanelli S, Indulsi F, Salomé B, Harari A, Romero $P$, et al. Dysregulated Innate Lymphocytes in Patients With Primary Antibody Deficiency Treated With Intravenous Immunoglobulin. J Investig Allergol Clin Immunol. 2017;27:394-6.

Manuscript received November 14, 2019; accepted for
publication January 8, 2020.

Victor Matheu

Módulo de Inmunodeficiencias Primarias Servicio de Alergología Hospital Universitario de Canarias Ctra Cuesta-Taco s/n 38320 La Cuesta, Tenerife, Spain E-mail: victor.matheu@gmail.com; alergia.tenerife1@gmail. com 